\title{
Fischer Type Log-Majorization of Singular Values on Partitioned Positive Semidefinite Matrices
}

\author{
Benju Wang $(\mathbb{D})$ and Yun Zhang \\ School of Mathematical Sciences, Huaibei Normal University, Huaibei 235000, China \\ Correspondence should be addressed to Yun Zhang; zhangyunmaths@163.com
}

Received 14 June 2021; Revised 23 July 2021; Accepted 16 August 2021; Published 31 August 2021

Academic Editor: Feliz Minhos

Copyright (C) 2021 Benju Wang and Yun Zhang. This is an open access article distributed under the Creative Commons Attribution License, which permits unrestricted use, distribution, and reproduction in any medium, provided the original work is properly cited.

In this paper, we establish a Fischer type log-majorization of singular values on partitioned positive semidefinite matrices, which generalizes the classical Fischer's inequality. Meanwhile, some related and new inequalities are also obtained.

\section{Introduction}

We denote by $M_{n, m}$ the vector space of all complex $n \times m$ matrices. In particular, when $n=m, M_{n}$ represents the set of square matrices of order $n$. Let $A$ and $B$ be Hermitian matrices. We use the notation $A \leq B$ or $B \geq A$ to mean that $B-A$ is positive semidefinite. Particularly, $B \geq 0 \quad(B>0)$ means that $B$ is positive semidefinite ( $B$ is positive definite). The singular values of $A \in M_{n}$ are the nonnegetive square roots of the eigenvalues of $A^{*} A$. We denote that $s_{j}(A)$ is the $j$ th largest singular value of $A \in M_{n}$ and we denote $s(A)=$ $\left(s_{1}(A), s_{2}(A), \cdots, s_{n}(A)\right)$. Denote by $\|\cdot\|_{\infty}$ the spectral norm. For $A \in M_{n}$, it is evident that $\|A\|_{\infty}=s_{1}(A)$.

We rearrange the components of $x=\left(x_{1}, x_{2}, \cdots, x_{n}\right) \in \mathbb{R}^{n}$ in decreasing order as $x_{[1]} \geq x_{[2]} \geq \cdots \geq x_{[n]}$. Let $x=\left(x_{1}, \cdots\right.$, $\left.x_{n}\right), y=\left(y_{1}, \cdots, y_{n}\right) \in \mathbb{R}^{n}$. If

$$
\sum_{i=1}^{k} x_{[i]} \leq \sum_{i=1}^{k} y_{[i]}, k=1,2, \cdots, n
$$

then we say that $x$ is weakly majorized by $y$ and denotes $x \prec_{\omega} y$. If $x \prec_{\omega} y$ and $\sum_{i=1}^{n} x_{i}=\sum_{i=1}^{n} y_{i}$, then we say that $x$ is majorized by $y$ and denotes $x<y$. Let nonnegative vectors $x, y \in \mathbb{R}_{+}^{n}$. If

$$
\prod_{i=1}^{k} x_{[i]} \leq \prod_{i=1}^{k} y_{[i]}, k=1,2, \cdots, n
$$

then we say that $x$ is weakly $\log$-majorized by $y$ and denotes $x \prec_{\omega \log } y$. If $x \prec_{\omega \log } y$ and $\prod_{i=1}^{n} x_{i}=\prod_{i=1}^{n} y_{i}$, then we say that $x$ is log-majorized by $y$ and denotes $x \prec_{\log } y$.

A norm on $M_{n}$ is called unitarily invariant if $\|U A V\|=$ $\|A\|$ for any $A \in M_{n}$ and any unitary $U, V \in M_{n}$. For $A \in$ $M_{n}$ and $1 \leq k \leq n$, the $k$ th compound matrix of $A$ is denoted by $C_{k}(A)$. We list one of the useful properties on compound matrices: for $A, B \in M_{n}, C_{k}(A B)=C_{k}(A) C_{k}(B)$. A series of properties of compound matrices can be seen in [1-3]. A complex matrix $C$ is called contraction if $C^{*} C \leq I$ or equivalently $s_{1}(C) \leq 1$. We denote the block matrix

$$
\left(\begin{array}{ll}
A & 0 \\
0 & C
\end{array}\right)
$$

by $A \oplus C$.

The well-known Fischer's inequality for determinant on the partitioned positive semidefinite matrix is the following. Let

$$
H=\left(\begin{array}{cc}
A & B \\
B^{*} & C
\end{array}\right) \geq 0 \text {, with } A \in M_{p} \text { and } C \in M_{q} .
$$


Then

$$
\operatorname{det}(H) \leq \operatorname{det}(A) \operatorname{det}(C)=\operatorname{det}(A \oplus C) .
$$

Let the eigenvalues of $A \in M_{n}$ be $\lambda_{1}, \lambda_{2}, \cdots, \lambda_{n}$ with $\left|\lambda_{1}\right|$ $\geq\left|\lambda_{2}\right| \geq \cdots \geq\left|\lambda_{n}\right|$. Weyl [4] proved that $\left\{\left|\lambda_{i}\right|\right\}_{i=1}^{n} \prec_{\log } s(A)$. Since for positive semidefinite matrices, singular values and eigenvalues are the same. Fischer's inequality can be rewritten as the following.

$$
\begin{aligned}
\operatorname{det}(H) & =\prod_{i=1}^{p+q} s_{i}(H) \leq \prod_{i=1}^{p} s_{i}(A) \prod_{i=1}^{q} s_{i}(C) \\
& =\prod_{i=1}^{p+q} s_{i}(A \oplus C)=\operatorname{det}(A \oplus C) .
\end{aligned}
$$

The motivation of this paper is to give the logmajorization relationship between the singular values of partitioned positive semidefinite matrix and its main diagonal matrix, which generalizes classical Fischer's inequality. In addition, we will also establish some singular value inequalities between partitioned positive semidefinite matrix and its main diagonal matrix.

\section{Main Results}

First, we list some lemmas that are used in our proofs.

Lemma 1 (Zhan [1], p. 71). Let $C$ be a complex matrix (not necessary square). Then, $C$ is a contraction if and only if

$$
\left(\begin{array}{cc}
I & C \\
C^{*} & I
\end{array}\right) \geq 0
$$

Lemma 2 (Zhan ([1], Theorem 3.34).

$$
\left(\begin{array}{ll}
A & B \\
B^{*} & C
\end{array}\right) \geq 0
$$

if and only if $A \geq 0, C \geq 0$, and there exists a contraction $W$ such that $B=A^{1 / 2} W C^{1 / 2}$.

Lemma 3 (Zhan [1], p. 80). If the singular values of $A \in M_{n}$ are $s_{1}, \cdots, s_{n}$, then the eigenvalues of

$$
\varphi(A):=\left(\begin{array}{cc}
0 & A \\
A^{*} & 0
\end{array}\right),
$$

are $s_{1}, \cdots, s_{n},-s_{n}, \cdots,-s_{1}$.
More general, by Jordan-Wielandt theorem ([5], Theorem 4.2), for $A \in M_{p, q}(p \geq q)$, the matrix

$$
\left(\begin{array}{cc}
0 & A \\
A^{*} & 0
\end{array}\right),
$$

has eigenvalues $\pm s_{i}, 0, \cdots, 0^{p-q \text { times }}, i=1, \cdots, q$.

Applying Lemma 3, we obtain the following.

Lemma 4. Let $W \in M_{p, q}(p \geq q)$ be the contractive matrix. Then,

$$
\begin{aligned}
s\left(\begin{array}{cc}
I & W \\
W^{*} & I
\end{array}\right) & =s(I+\varphi(W)) \\
& =\left(1+s_{1}, \cdots, 1+s_{q}, 1, \cdots, 1,1-s_{q}, \cdots, 1-s_{1}\right),
\end{aligned}
$$

where $s_{i}$ is the ith largest singular value of $W$.

Proof. $W$ is a contractive matrix if and only if

$$
\left(\begin{array}{cc}
I & W \\
W^{*} & I
\end{array}\right) \geq 0
$$

For positive semidefinite matrices, singular values and eigenvalues are the same. Note that

$$
\left(\begin{array}{cc}
I & W \\
W^{*} & I
\end{array}\right)=\left(\begin{array}{ll}
I & 0 \\
0 & I
\end{array}\right)+\left(\begin{array}{cc}
0 & W \\
W^{*} & 0
\end{array}\right),
$$

by Lemma 3 and spectral mapping theorem, this completes the proof.?

Next, we will establish the log-majorization relationship between the singular values of partitioned positive semidefinite matrix and its main diagonal matrix.

Theorem 5. Let

$$
\left(\begin{array}{cc}
A & B \\
B^{*} & C
\end{array}\right) \in M_{p+q}
$$

be positive semidefinite matrix with $A \in M_{p}, C \in M_{q}(p \geq q)$. Then, there exists a contractive matrix $W \in M_{p, q}$ such that

$$
s\left(\begin{array}{cc}
A & B \\
B^{*} & C
\end{array}\right) \prec_{\log }\left\{s_{i}(I+\varphi(W)) s_{i}(A \oplus C)\right\}_{i=1}^{p+q} .
$$

Proof. Applying Lemma 2, there exists a contractive matrix $W$ such that $B=A^{1 / 2} W C^{1 / 2}$. Then, 


$$
\left(\begin{array}{cc}
A & B \\
B^{*} & C
\end{array}\right)=\left(\begin{array}{cc}
A^{1 / 2} & 0 \\
0 & C^{1 / 2}
\end{array}\right)^{*}\left(\begin{array}{cc}
I_{p} & W \\
W^{*} & I_{q}
\end{array}\right)\left(\begin{array}{cc}
A^{1 / 2} & 0 \\
0 & C^{1 / 2}
\end{array}\right) .
$$

Denote

$$
\begin{aligned}
& H=\left(\begin{array}{cc}
A & B \\
B^{*} & C
\end{array}\right), Q=\left(\begin{array}{cc}
I_{p} & W \\
W^{*} & I_{q}
\end{array}\right), \\
& P=\left(\begin{array}{cc}
A^{1 / 2} & 0 \\
0 & C^{1 / 2}
\end{array}\right), T=A \oplus C .
\end{aligned}
$$

The equality (16) can be rewritten as $H=P^{*} Q P$. Using compound matrices and the fact that for any square matrix $A,\|A\|_{\infty}^{2}=\left\|A^{*} A\right\|_{\infty}$, for $1 \leq k \leq p+q$, we have

$$
\begin{aligned}
\prod_{j=1}^{k} s_{j}(H) & =\prod_{j=1}^{k} s_{j}\left(P^{*} Q P\right)=s_{1}\left[C_{k}\left(P^{*} Q P\right)\right] \\
& =s_{1}\left[C_{k}\left(P^{*}\right) C_{k}(Q) C_{k}(P)\right] \\
& =\left\|C_{k}\left(P^{*}\right) C_{k}(Q) C_{k}(P)\right\|_{\infty} \\
& \leq\left\|C_{k}\left(P^{*}\right)\right\|_{\infty}\left\|C_{k}(Q)\right\|_{\infty}\left\|C_{k}(P)\right\|_{\infty} \\
& =\left\|C_{k}\left(P^{*}\right)\right\|_{\infty}^{2}\left\|C_{k}(Q)\right\|_{\infty} \\
& =\left\|C_{k}\left(P^{*}\right) C_{k}(P)\right\|_{\infty}\left\|C_{k}(Q)\right\|_{\infty} \\
& =\left\|C_{k}\left(P^{*} P\right)\right\|_{\infty}\left\|C_{k}(Q)\right\|_{\infty} \\
& =\left\|C_{k}(T)\right\|_{\infty}\left\|C_{k}(Q)\right\|_{\infty} \\
& =\prod_{j=1}^{k} s_{j}(T) s_{j}(Q) .
\end{aligned}
$$

In addition, for $k=p+q$, we have

$$
\begin{aligned}
\operatorname{det}(H) & =\prod_{j=1}^{p+q} s_{j}(H)=\operatorname{det}\left(P^{*} Q P\right)=\operatorname{det}(Q T) \\
& =\prod_{j=1}^{p+q} s_{j}(I+\varphi(W)) \prod_{i=1}^{p+q} s_{i}(A \oplus C) \\
& =\prod_{j=1}^{q}\left(1-s_{j}(W)^{2}\right) \prod_{i=1}^{p+q} s_{i}(A \oplus C),
\end{aligned}
$$

where the last equality is due to Lemma 4, this completes the proof.?

\section{Corollary 6. Let}

$$
H=\left(\begin{array}{cc}
A & B \\
B^{*} & C
\end{array}\right) \in M_{p+q}
$$

be positive semidefinite matrix with $A \in M_{p}, C \in M_{q}(p \leq q)$. Then, there exists a contractive matrix $W \in M_{p, q}$ such that

$$
\operatorname{det}(H)=\prod_{j=1}^{q}\left(1-s_{j}(W)^{2}\right) \operatorname{det}(A \oplus C) .
$$

Remark 7. From the equality (21), we can see that $\operatorname{det}(H)$ $\leq \operatorname{det}(A \oplus C)$. The equality holds if and only if $s(W)=0$, i.e., $W=0$; it also implies that $B=0$.

Corollary 8. Let

$$
H=\left(\begin{array}{cc}
A & B \\
B^{*} & C
\end{array}\right) \in M_{p+q}
$$

be positive semidefinite matrix with $A \in M_{p}, C \in M_{q}$. Then, there exists a contractive matrix $W \in M_{p, q}$ such that

$$
\left\|\left(\begin{array}{ll}
A & B \\
B^{*} & C
\end{array}\right)\right\| \leq\left(1+s_{1}(W)\right) \cdot\|A \oplus C\| .
$$

for all unitarily invariant norms $\|\cdot\|$.

Proof. Recall that weak log-majorization is stronger than weak majorization, i.e., $x \prec_{\omega} \log y$ implies $x \prec_{\omega} y$. By Theorem 5 , we have

$$
\begin{aligned}
& \left(s_{1}(H), \cdots, s_{p+q}(H)\right) \\
& \quad{ }_{\omega}\left(\left(1+s_{1}(W)\right) s_{1}(A \oplus C), \cdots,\left(1-s_{1}(W)\right) s_{p+q}(A \oplus C)\right) .
\end{aligned}
$$

It is clear that

$$
\begin{aligned}
& \left(\left(1+s_{1}(W)\right) s_{1}(A \oplus C), \cdots,\left(1-s_{1}(W)\right) s_{p+q}(A \oplus C)\right) \\
& \leq\left(\left(1+s_{1}(W)\right) s_{1}(A \oplus C), \cdots,\left(1+s_{1}(W)\right) s_{p+q}(A \oplus C)\right) .
\end{aligned}
$$

Therefore, we have

$$
\begin{aligned}
& \left(s_{1}(H), \cdots, s_{p+q}(H)\right) \\
& \quad{ }_{\omega}\left(\left(1+s_{1}(W)\right) s_{1}(A \oplus C), \cdots,\left(1+s_{1}(W)\right) s_{p+q}(A \oplus C)\right) .
\end{aligned}
$$

Using the Fan Dominance Principle (see [6]), this completes the proof.?

In the following section, we will establish some singular value inequalities between partitioned positive semidefinite matrix and its main diagonal matrix. The following wellknown result is due to Ky Fan [1].

Lemma 9. Let $A, B \in M_{n}, 1<i, j \leq n, i+j-1 \leq n$. Then,

$$
s_{i+j-1}(A B) \leq s_{i}(A) s_{j}(B) .
$$


In particular,

$s_{j}(A B) \leq s_{1}(A) s_{j}(B), s_{j}(A B) \leq s_{1}(B) s_{j}(A), j=1, \cdots, n$.

Theorem 10. Let

$$
\left(\begin{array}{ll}
A & B \\
B^{*} & C
\end{array}\right) \in M_{p+q}
$$

be positive semidefinite matrix with $A \in M_{p}, C \in M_{q}(p \geq q)$. Then, there exists a contractive matrix $W \in M_{p, q}$ such that

$$
s_{i}\left(\begin{array}{ll}
A & B \\
B^{*} & C
\end{array}\right) \leq\left(1+s_{1}(W)\right) s_{i}(A \oplus C), i=1, \cdots, p+q .
$$

In addition, for $1 \leq i \leq q$,

$$
s_{i}\left(\begin{array}{ll}
A & B \\
B^{*} & C
\end{array}\right) \leq\left(1+s_{i}(W)\right) s_{1}(A \oplus C),
$$

for $q+1 \leq i \leq q+p$,

$$
s_{i}\left(\begin{array}{cc}
A & B \\
B^{*} & C
\end{array}\right) \leq s_{1}(A \oplus C) .
$$

Proof.

$$
\begin{aligned}
s_{i}\left(\begin{array}{cc}
A & B \\
B^{*} & C
\end{array}\right) & =s_{i}\left[\left(\begin{array}{cc}
A^{1 / 2} & 0 \\
0 & C^{1 / 2}
\end{array}\right)^{*}\left(\begin{array}{cc}
I_{p} & W \\
W^{*} & I_{q}
\end{array}\right)\left(\begin{array}{cc}
A^{1 / 2} & 0 \\
0 & C^{1 / 2}
\end{array}\right)\right] \\
& \leq s_{i}\left(\begin{array}{cc}
A^{1 / 2} & 0 \\
0 & C^{1 / 2}
\end{array}\right) s_{1}\left[\left(\begin{array}{cc}
I_{p} & W \\
W^{*} & I_{q}
\end{array}\right)\left(\begin{array}{cc}
A^{1 / 2} & 0 \\
0 & C^{1 / 2}
\end{array}\right)\right] \\
& \leq s_{i}\left(\begin{array}{cc}
A^{1 / 2} & 0 \\
0 & C^{1 / 2}
\end{array}\right) s_{1}\left(\begin{array}{cc}
I_{p} & W \\
W^{*} & I_{q}
\end{array}\right) s_{i}\left(\begin{array}{cc}
A^{1 / 2} & 0 \\
0 & C^{1 / 2}
\end{array}\right) \\
& =s_{1}\left(\begin{array}{cc}
I_{p} & W \\
W^{*} & I_{q}
\end{array}\right) s_{i}\left(\begin{array}{cc}
A & 0 \\
0 & C
\end{array}\right) \\
& =\left(1+s_{1}(W)\right) s_{i}\left(\begin{array}{cc}
A & 0 \\
0 & C
\end{array}\right) .
\end{aligned}
$$

In (33), change the position of

$$
\left(\begin{array}{cc}
A^{1 / 2} & 0 \\
0 & C^{1 / 2}
\end{array}\right)
$$

and

$$
\left(\begin{array}{cc}
I_{p} & W \\
W^{*} & I_{q}
\end{array}\right)\left(\begin{array}{cc}
A^{1 / 2} & 0 \\
0 & C^{1 / 2}
\end{array}\right)
$$

By similar proof method, we can get the following inequality.

$$
s_{i}\left(\begin{array}{cc}
A & B \\
B^{*} & C
\end{array}\right) \leq s_{i}\left(\begin{array}{cc}
I & W \\
W^{*} & I
\end{array}\right) s_{1}(A \oplus C), i=1, \cdots, p+q .
$$

Using Lemma 4, we can divide into the following cases: Case $1.1 \leq i \leq q$. Then,

$$
s_{i}\left(\begin{array}{cc}
A & B \\
B^{*} & C
\end{array}\right) \leq\left(1+s_{i}(W)\right) s_{1}(A \oplus C) .
$$

Case 2. $q+1 \leq i \leq p+q$. Then,

$$
s_{i}\left(\begin{array}{cc}
A & B \\
B^{*} & C
\end{array}\right) \leq s_{1}(A \oplus C) .
$$

This completes the proof.?

The following theorem is proved by using the result given by Hirzallah and Kittaneh [7].

Lemma 11. Let $A, B \in M_{n}$ and $0<\alpha<1$. Then,

$$
s_{j}(\alpha A+(1-\alpha) B) \leq s_{j}(A \oplus B), j=1, \cdots, 2 n .
$$

In particular, letting $\alpha=1 / 2$, we have

$$
s_{j}\left(\frac{A+B}{2}\right) \leq s_{j}(A \oplus B), j=1, \cdots, 2 n .
$$

Theorem 12. Let

$$
\left(\begin{array}{cc}
A & B \\
B^{*} & C
\end{array}\right) \in M_{p+q}
$$

be positive semidefinite matrix with $A \in M_{p}, C \in M_{q}(p \geq q)$. Then, for $j=1, \cdots, p+q$,

$$
s_{j}\left(\begin{array}{cc}
A & 0 \\
0 & C
\end{array}\right) \leq s_{j}\left(\left(\begin{array}{cc}
A & B \\
B^{*} & C
\end{array}\right) \oplus\left(\begin{array}{cc}
A & B \\
B^{*} & C
\end{array}\right)\right) .
$$

Proof. Since

$$
\left(\begin{array}{cc}
I_{p} & 0 \\
0 & -I_{q}
\end{array}\right)\left(\begin{array}{cc}
A & B \\
B^{*} & C
\end{array}\right)\left(\begin{array}{cc}
I_{p} & 0 \\
0 & -I_{q}
\end{array}\right)=\left(\begin{array}{cc}
A & -B \\
-B^{*} & C
\end{array}\right),
$$

we have

$$
s_{j}\left(\begin{array}{cc}
A & B \\
B^{*} & C
\end{array}\right)=s_{j}\left(\begin{array}{cc}
A & -B \\
-B^{*} & C
\end{array}\right) .
$$


Meanwhile

$$
\left(\begin{array}{ll}
A & 0 \\
0 & C
\end{array}\right)=\frac{1}{2}\left(\begin{array}{ll}
A & B \\
B^{*} & C
\end{array}\right)+\frac{1}{2}\left(\begin{array}{cc}
A & -B \\
-B^{*} & C
\end{array}\right) .
$$

Applying Lemma 11 to (45), we have

$$
\begin{aligned}
s_{j}\left(\begin{array}{ll}
A & 0 \\
0 & C
\end{array}\right) & \leq s_{j}\left(\left(\begin{array}{ll}
A & B \\
B^{*} & C
\end{array}\right) \oplus\left(\begin{array}{cc}
A & -B \\
-B^{*} & C
\end{array}\right)\right) \\
& =s_{j}\left(\left(\begin{array}{ll}
A & B \\
B^{*} & C
\end{array}\right) \oplus\left(\begin{array}{cc}
A & B \\
B^{*} & C
\end{array}\right)\right) .
\end{aligned}
$$

This complete the proof.?

Remark 13. One may ask whether the inequality holds

$$
s_{j}\left(\begin{array}{ll}
A & 0 \\
0 & C
\end{array}\right) \leq s_{j}\left(\begin{array}{cc}
A & B \\
B^{*} & C
\end{array}\right), j=1, \cdots, p+q .
$$

But it needs not be true. For example, setting

$$
\left(\begin{array}{ll}
A & B \\
B^{*} & C
\end{array}\right)=\left(\begin{array}{ll}
1 & 1 \\
1 & 1
\end{array}\right) \geq 0,\left(\begin{array}{ll}
A & 0 \\
0 & C
\end{array}\right)=\left(\begin{array}{ll}
1 & 0 \\
0 & 1
\end{array}\right),
$$

Then,

$$
s\left(\begin{array}{ll}
1 & 1 \\
1 & 1
\end{array}\right)=(2,0), s\left(\begin{array}{ll}
1 & 0 \\
0 & 1
\end{array}\right)=(1,1) .
$$

Obviously,

$$
s_{2}\left(\begin{array}{ll}
1 & 1 \\
1 & 1
\end{array}\right)=0<1=s_{2}\left(\begin{array}{ll}
1 & 0 \\
0 & 1
\end{array}\right) .
$$

\section{Data Availability}

The data used to support the findings of this study are included within the article.

\section{Conflicts of Interest}

The author declares that he have no conflicts of interest.

\section{Authors' Contributions}

The author reads and approves the final manuscript.

\section{Acknowledgments}

The work was supported by the National Science Foundation of Anhui Higher Education Institutions of China (KJ2019A0588 and KJ2020ZD008).

\section{References}

[1] X. Zhan, Matrix Theory, Graduate Studies in Mathematics, Mathematical Society, Providence, RI, USA, 2013.

[2] R. Bhatia, Matrix Analysis, Springer-Verlag, 1997.

[3] R. A. Horn and C. R. Johnson, Matrix Analysis, Cambrige University press, 2013.

[4] H. Weyl, "Inequalities between the two kinds of eigenvalues of a linear transformation," Proceedings of the National Academy of Sciences of the United States of America, vol. 35, no. 7, pp. 408$411,1949$.

[5] G. W. Stewart and J.-g. Sun, Matrix Perturbation Theory, Computer Science and Scientific Computing, 1990.

[6] K. Fan, "Maximum properties and inequalities for the eigenvalues of completely continuous operators," Proceedings of the National Academy of Sciences of the United States of America, vol. 37, no. 11, pp. 760-766, 1951.

[7] O. Hirzallah and F. Kittaneh, "Inequalities for sums and direct sums of Hilbert space operators," Linear Algebra and its Applications, vol. 424, no. 1, pp. 71-82, 2007. 